\title{
Seismic exclusions and re-entry from a risk perspective
}

\author{
SR Tierney Australian Centre for Geomechanics, The University of Western Australia, Australia \\ KR Woodward Australian Centre for Geomechanics, The University of Western Australia, Australia
}

J Wesseloo Australian Centre for Geomechanics, The University of Western Australia, Australia

\begin{abstract}
Exposure to seismic hazard in mines is controlled through various evacuation, exclusion and re-entry procedures. The aim of exposure management procedures is to tactically reduce the safety risk by removing personnel from work areas during periods of elevated seismic hazard. Given that risk assessment is based on exposure, the design of exposure management procedures must also be risk-based.

In practice, the decision to re-enter a workplace after an exclusion is generally only made based on an assessment of the seismic hazard, often using previously defined levels of tolerable seismic activity rates. The definition of tolerable seismic hazard, in the context of re-entry, is seldom quantitatively assessed based on risk. In order to move towards a comprehensive seismic risk management strategy, design methodologies must be able to quantify the impact of different exclusion and re-entry practices on risk. The appropriate re-entry practice can then be selected given the defined risk-based design acceptance criteria.
\end{abstract}

There is still a long way to go before the risk-based design framework for exposure management procedures is complete. This paper reviews the current state of design of exposure management controls in the context of risk and discusses several important areas for further research.

Keywords: seismicity, risk-based design, exposure, exclusions, re-entry, seismic hazard

\section{Introduction}

The risk associated with mining-induced seismicity, or dynamic rock mass failure, is well-known in the international mining industry particularly in deep, hard rock, underground mines. Considerable research and resources have been dedicated to improved understanding and management of this risk. While the number of injuries and fatalities have decreased in recent decades, rock related accidents are still a regular occurrence around the world. These accidents have severe economic, psychological and sociological consequences for individuals, organisations and communities.

Seismic risk in mines is managed through a combination of control measures such as dynamic ground support systems, limiting personnel exposure, and favourable mine layouts and sequences. These control measures are implemented based on a risk assessment, at least implicitly. A seismic risk assessment requires an understanding of the seismic hazard, the vulnerability of excavations, and the exposure of the workforce during mining operations (Figure 1).

Exposure to seismic hazard in mines is reduced through various evacuation, exclusion and re-entry procedures. The aim of exposure management procedures is to tactically reduce the safety risk by removing personnel from work areas during periods of elevated seismic hazard.

Exposure is a core component of risk and, therefore, the design of exposure management controls should also be risk-based. This paper discusses the design of seismic exposure management procedures from a risk perspective in the context of a comprehensive seismic risk management strategy for underground mines. 


\section{$2 \quad$ Risk-based design and management}

A civil engineer might design their building or bridge based on an acceptance criterion in terms of Factor of Safety (FS) or Probability of Failure (PF). Both FS and PF are design acceptance criteria that focus on stability. Structures for public use generally have severe consequences in the event of failure. In mining, the severity of the consequences to a rockfall are wide ranging. A rockfall might occur in a closed off area that is no longer required or a rockfall might have catastrophic consequences. Joughin et al. (2016) points out 'in mining, failure with no adverse consequence is preferable to unnecessary stability at a high cost'. This makes risk (the combination of PF and consequences) the ideal design acceptance criterion for mining.

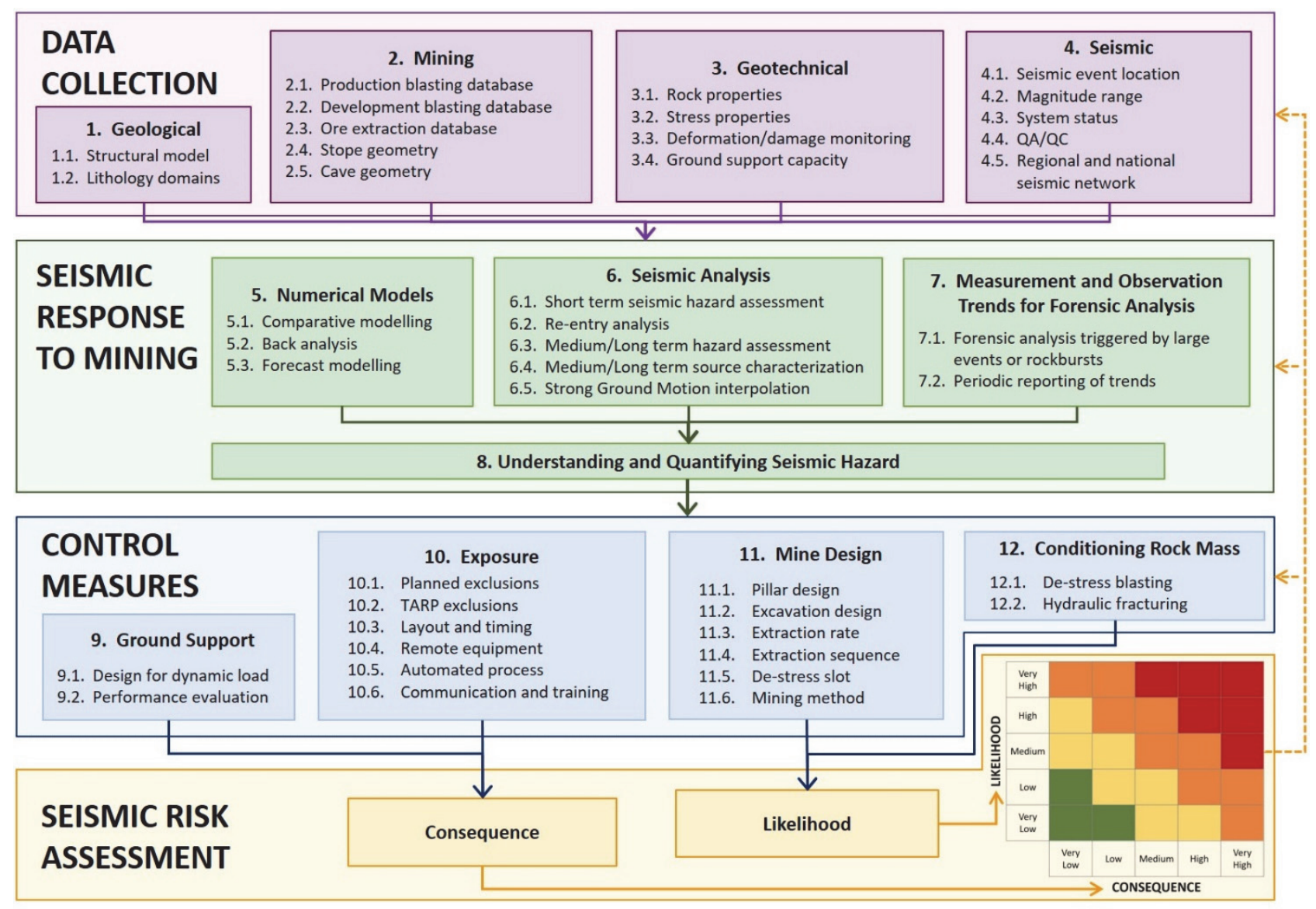

Figure 1 A flowchart of a seismic risk management process for underground mines (Potvin et al. 2018)

Risk management and risk-based design can be related to different types of risk such as economic, safety and health, environmental and reputational. It is usually easier to talk about economic risk than risks associated with the safety and health of people. Companies are generally more comfortable discussing the difference between high-risk, high-return endeavours and low-risk, low-return endeavours since the shareholder value is dependent on the probability of actually realising the return, which is rarely the highest risk option. The decision-making behind issues related to safety and health is much more difficult, and companies usually refrain from explicit policies regarding tolerable risk criteria for health and safety incidents. Nevertheless, risk is accepted in everyday life and stopping discussion of these topics does not help in improving the quantification and management of risk in the workplace.

Historically, mining was a high-risk industry that had an acceptance of casualties associated with working underground. 'Accidents just happen' was an excuse used defensively by mining operators. A recent example being the incident where 29 miners were killed by an explosion in West Virginia in 2010. The CEO told reporters that he believed that "the physics of natural law and God trump whatever man tries to do" (Kirsch et al. 2014). Such attitudes, though, are generally something of the distant past, and safety in corporate 
mines is generally comparable to other industries. Figure 2 presents data published by Safe Work Australia (2016) showing Australian workplace fatalities for difference industries (fatalities normalised per 100,000 workers). It is increasingly recognised that to retain their social licence to operate, mining companies should be committed to a policy of zero harm, where every worker returns home safe and healthy every day. This attitude has no doubt contributed to the improved safety performance at mines.

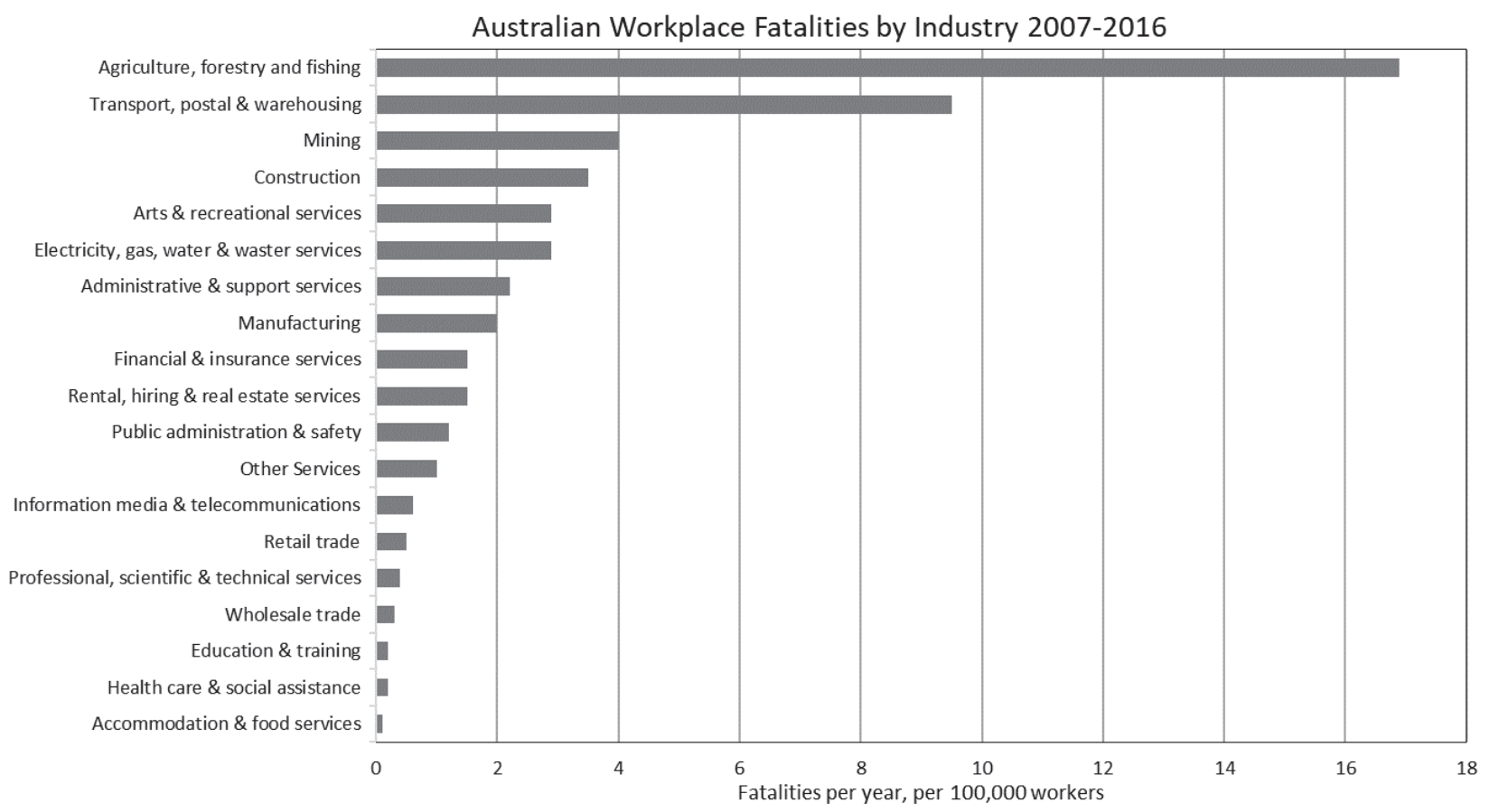

Figure 2 Australian worker fatalities by industry. 10 years data from 2007 to 2016. Annual fatality rate plotted per 100,000 workers in the industry (Safe Work Australia 2016)

While the mission of zero harm has undoubtedly been a positive driving force for the significant improvements to health and safety within the mining industry, this fact does not exempt the policy from the acknowledgement and discussion of its negative implications for risk management. A zero harm policy increases the commitment to safety for some people, but it also leads to disinterest in others who consider it an unfeasible goal. Sherratt (2014) explored the consequences of safety programs targeting zero harm in the UK construction industry. He raised concerns that the corporate level and site level employees had differing visions of how the 'zero' target works in practice. The 'zero' mentality tends to shut down the discussion of practical risk management. In fact, mine management would rarely utter the terms 'probability of fatality' or 'acceptable risk'. Eliminating discussion does not eliminate the reality that there are accepted risks in everyday life. It is true that no moral person looks at a fatal car accident and considers it 'acceptable' but some fatalities in car accidents are 'accepted' by the government and by society.

Another consequence of an emphasis on zero harm is that companies tend to focus on eradicating the lowest impact (and more numerous) incidents. This does not align with the prioritisation of risk management and may dilute the control of rare incidents that are more severe (Wentzel et al. 2012). This is often justified by citing the Heinrich (or Bird) safety triangle; i.e. the premise that there is a stable ratio between the number of severe-rare and minor-frequent incidents but this notion has been broadly invalidated (Anderson \& Denkl 2010; Gallivan et al. 2008; Manuele 2011). Incidents that are high-frequency, low-severity constitute the majority of reported incident statistics and, therefore, naturally demand more focus from management. Unfortunately, there are a number of cognitive biases embedded in human psychology that limit our motivation for managing risks that are rare but also high severity. These biases are essentially identical to those discussed by Yudkowsky (2008) in the context of managing existential risks such as climate change and asteroids. 
Zero risk is an impossibility and, therefore, engineering design cannot be for a state of zero risk. A strategic company target of zero harm may be appropriate, but this should not be mistaken for a design aim, and it is not useful for site management to determine acceptable limits of risk. Criteria for tolerable levels of risk are essential for design and risk management, otherwise there will be confusion and misalignment between engineers, managers and the authorities (Joughin 2011). In most cases, the technical specialist is not qualified or is not responsible for determining the tolerable risk level. The technical specialist must find effective means of communicating the risk, and mine management must not abdicate responsibility for determining what is acceptable (Terbrugge et al. 2006).

Several authors have discussed the definition of tolerable levels of safety risk where the accepted risk of fatalities is not zero (e.g. Bohnenblust 1998; Finlay \& Fell 1997; Higson 1989; Jonkman et al. 2003; Joughin et al. 2012; Melchers 2001; Stacey et al. 2007; Terbrugge et al. 2006; Vrijling et al. 1995, 1998; Wesseloo \& Joughin 2019). More specifically, Wesseloo and Joughin (2019) define individual and corporate safety risk acceptance levels for underground mines in Australia. This type of work does not seek to define a level of risk to impose on society, rather attempt to quantify the level of risk that is already accepted by society. Accepted risk criteria are based on the quantification of the risk that people are exposed to on a regular basis in the course of daily life.

The as low as reasonably practicable (ALARP) principle is built into the risk management legislation in many western countries. This concept is illustrated in Figure 3. Risks are to be reduced until both of the following conditions are met:

1. The overall level of risk is tolerable.

2. A further reduction would have a disproportionate cost associated with the benefits gained.

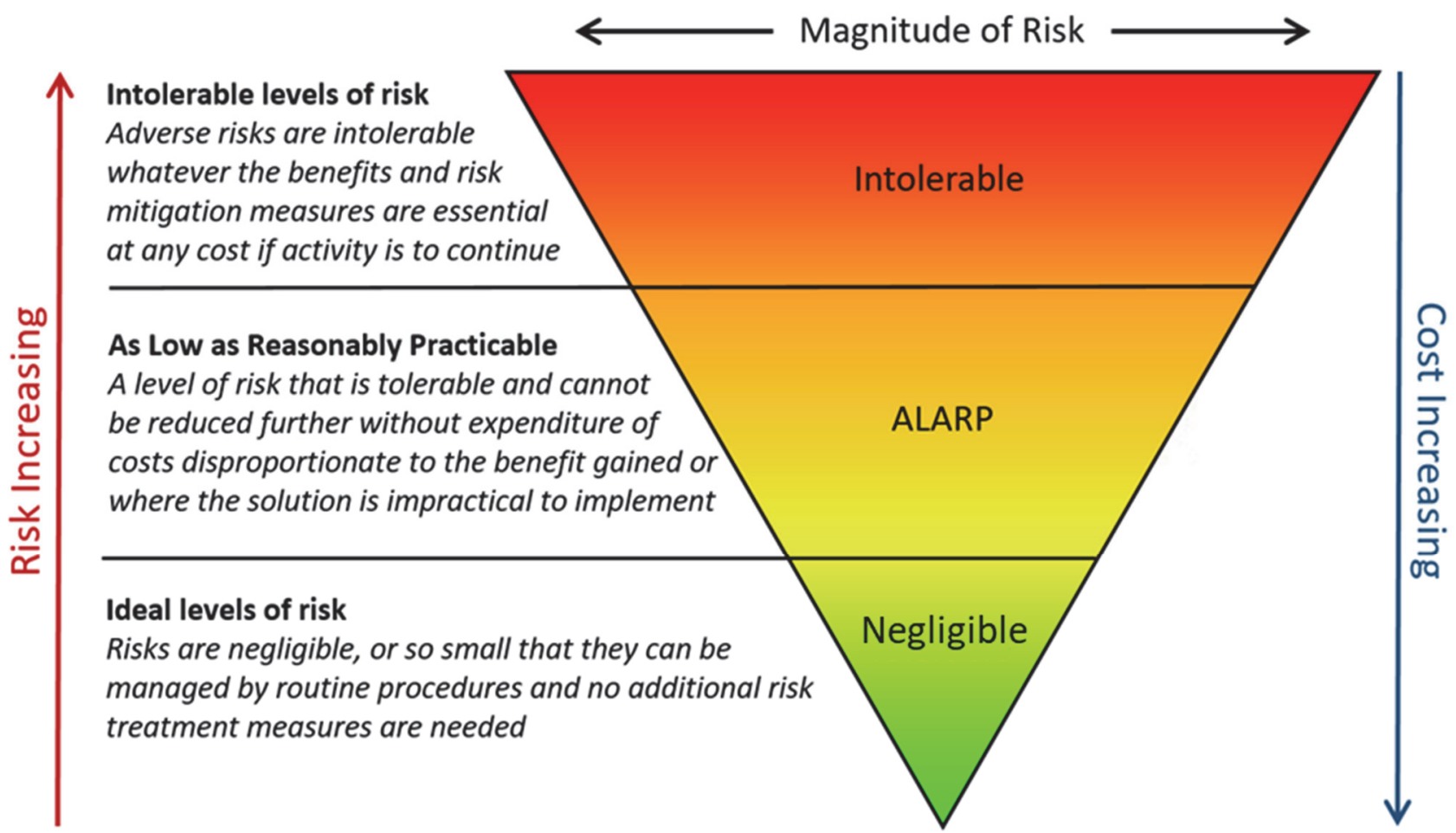

Figure 3 Illustration of intolerable and negligible risk levels and the ALARP principle. Costs increase in the downward direction, risk increases in the upward direction, and the magnitude of the risk is depicted as the width of the inverse pyramid (Talbot 2014).

While there is a reasonable body of reference material to adequately define negligible and intolerable risk levels, the supporting literature behind the ALARP safety risk level is much thinner. The ALARP principle by definition requires the comparison of the cost and the benefit of the potential risk reduction. This is easier 
when assessing economic risk since the costs and benefits are both in financial units. There has been previous work to assign a monetary value to a human life or injury, but this is considered by most to be morally indefensible. As a result, design criteria for economic and safety risk are considered separately. It is still unclear how the mechanics of the ALARP principle integrate into a quantified risk-based engineering design methodology given the costs and benefits of control measures to manage safety risk are in different, non-convertible units.

Effectively assessing and communicating risk is not an easy task, and there are very few cases where the risks associated with underground mining have been quantified in literature; particularly health and safety risk. Most mines perform qualitative assessments using risk matrices like the example in Figure 4. This requires considerable engineering judgement and estimation. The risk is generally assessed for a particular job or list of tasks. Control measures are discussed that will reduce the risk in a similar spirit as the ALARP principle. An intolerable level of risk may be specified as the 'very high' category at some mines.

A drawback when using risk matrices to assess the safety risk from a rockfall is that most scenarios fit into the rare and severe categories. This makes it difficult to distinguish between different scenarios and risk management controls. Risk matrices are a blunt instrument and should only be used for short-term tactical decisions. Risk-based design and strategic decisions require a more in-depth approach.

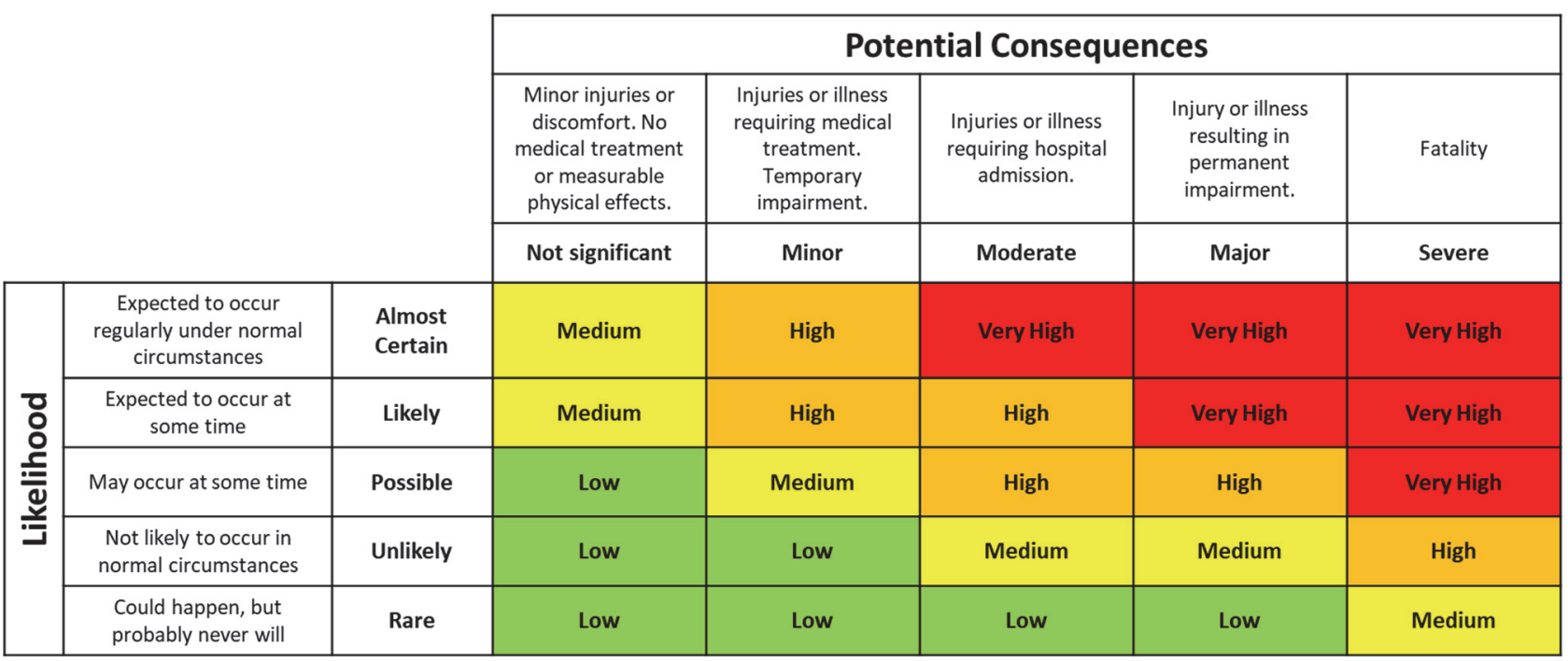

Figure 4 Example of a safety and health risk matrix for qualitative assessment (Cohen 2015)

Fault and event trees are a useful tool to illustrate and evaluate risk for design and strategic decisions. Figure 5 illustrates the connection between PF and safety risk for an individual (Figure 5(a)) and for corporate/collective risk (Figure $5($ b)). In the event of a rockfall, the safety risk can be evaluated by estimating:

1. The effectiveness of exposure management procedures.

2. Given the exposure management failed, the likelihood of spatial and temporal coincidence between personnel and the rockfall.

3. Given the spatial and temporal coincidence, the likelihood of injury and fatality.

It is important to consider both corporate and individual risk, as there are cases where either could be above the tolerable risk threshold. Individual risk may be higher than corporate risk if a single person works all day in a high hazard area while the majority of the workforce works in a low-hazard location. In a high-hazard area, if personnel are rotated every two hours, the individual risk is limited. However, the corporate risk is the sum of each individual risk and is, therefore, still high.

The conversion between PF and risk is heavily dependent on the mining methods and layout, the nature of the work, and the exposure management procedures. Owen (2004) analysed the exposure of personnel to 
geotechnical hazards in underground mining and developed an exposure model to quantify operational risk. The exposure model incorporates a profile of a mine's workforce and fleet, materials-handling methods, working locations, and other activities related to production, development, services and technical support. Clearly, in order to quantify the safety risk, it is critical to understand and quantify the effectiveness of exposure management protocols. Only then can the risk be compared with tolerable levels.

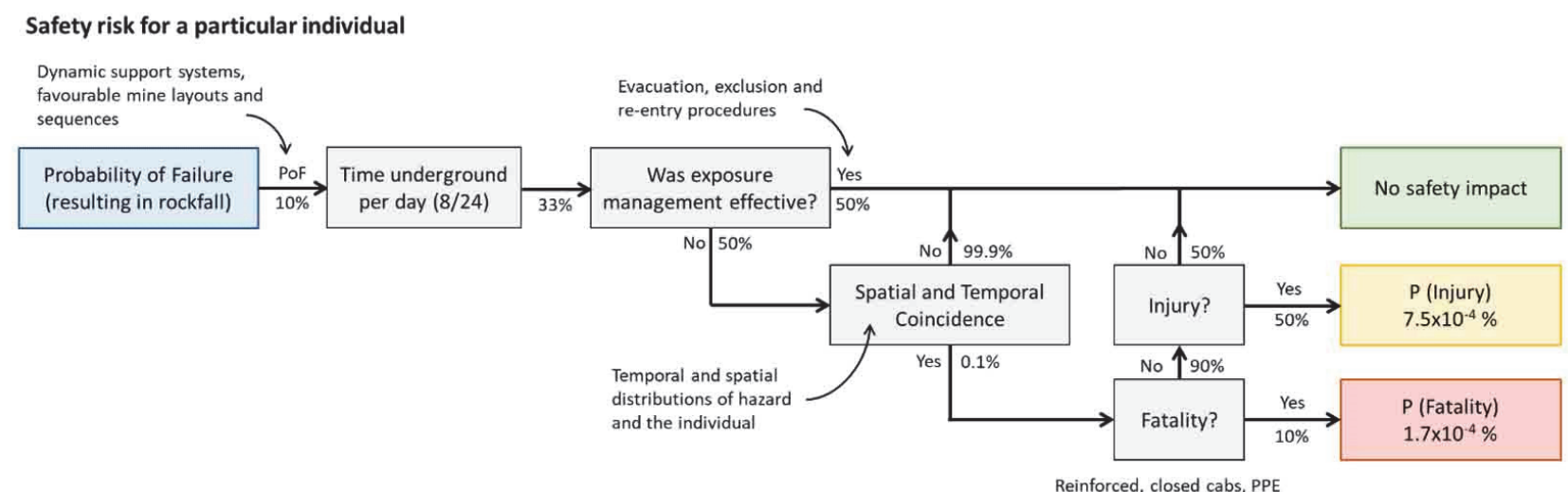

(a)

Safety risk for ANY single person or multiple persons (corporate or collective risk)

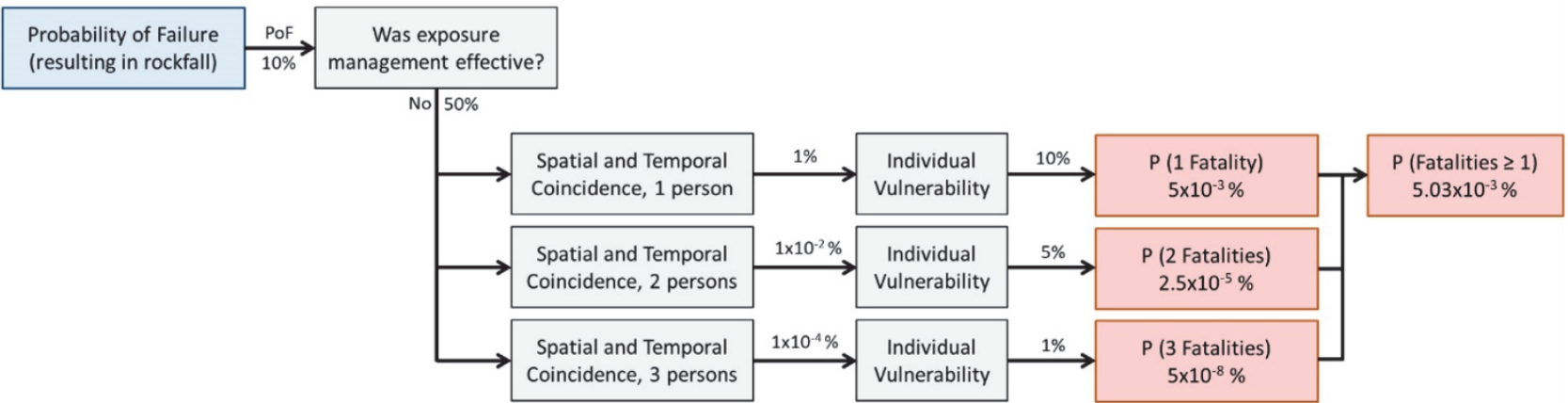

(b)

Figure 5 Illustration of the relationship between Probability of Failure and Probability of Fatality and the role of exposure management controls. Branch probabilities are nominal only; actual values would need to be associated with a spatial and temporal resolution. Fault trees for (a) Individual risk; (b) Corporate risk

\section{Seismic hazard}

The seismic hazard is a key influence on the PF (stability) of underground excavations and therefore on risk. The PF due to a rockburst is a function of the seismic hazard and the excavation vulnerability, which is influenced by the ground support system and site-specific geological and geometric conditions.

The assessment of seismic hazard requires an understanding of the spatial, frequency, and magnitude distributions of seismic events. These distributions vary over time due to variations in numerous causative factors such as principal stress conditions, mining geometry, rock mass characteristics, and blasting. Hence, seismic hazard should also be considered with respect to the nature of mining along with generalised geological and geotechnical information.

In theory, the decision to exclude and re-enter a work area is based on a risk assessment that considers the seismic hazard, the potential work activities and the vulnerability of the excavations. In practice, re-entry is generally only made based on an assessment of the current seismic hazard; often using previously defined levels of tolerable seismic activity rates. The focus on assessing the current seismic hazard is not without reason, provided the defined tolerable hazard levels consider any variability in excavation vulnerability 
between different areas. Seismic hazard is the most variable factor that influences excavation stability within re-entry time scales. Factors such as excavation geometry and ground support systems are planned in advance of mining and tend to be less variable over time.

Figure 6 illustrates the relationship between risk and seismic hazard that varies over time. Seismic hazard varies in space and time, and often increases rapidly after blasting or significant events. The risk for a given time window is proportional to the hazard state integrated over time. A very short spike in the instantaneous hazard state can mean very little in terms of the overall hazard in the long-term. In Figure 6(a), the average hazard is indicated as the constant hazard level that would result in an equivalent total risk over the time window.

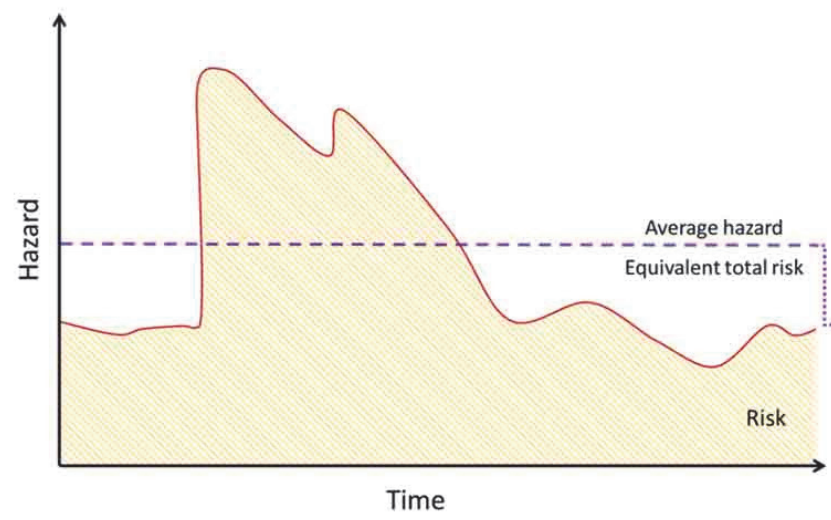

(a)

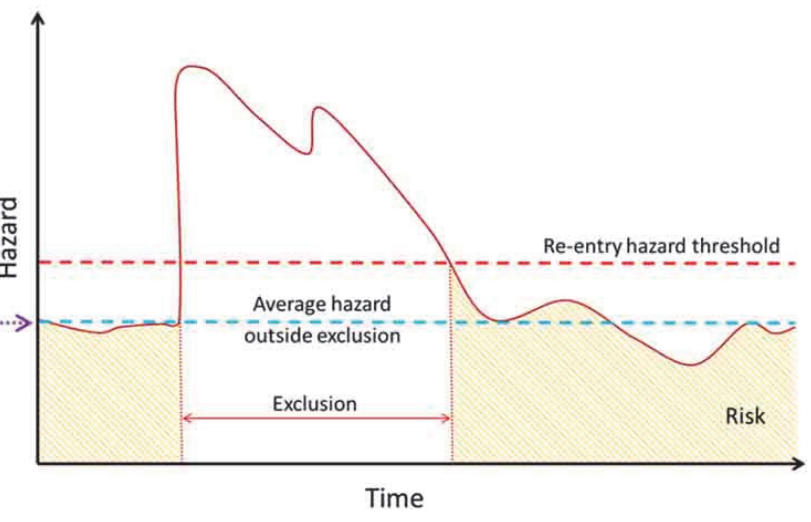

(b)

Figure 6 Illustration of hazard varying over time, and the effect of an exclusion based on a threshold hazard state. For a particular time period, the hazard integrated over time is proportional to risk. The average hazard is the constant hazard level that would result in an equivalent risk across (a) The entire time period; (b) The time outside exclusion

Figure 6(b) illustrates the effect on risk if an exclusion is initiated, and re-entry occurs based on a threshold hazard state (exclusion initiation may be based on a different criterion than re-entry). There is a distinction between the hazard threshold used for re-entry and the resulting average hazard outside exclusion. In the case of decreasing seismic hazard after a blast, the average hazard that is relevant to risk will generally be lower than the re-entry hazard threshold. The goal of an exclusion is to reduce risk by tactically reducing exposure during periods of high hazard. For a risk-based design of exclusion and re-entry protocols, an effective means of estimating seismic hazard is essential, both for the short-term re-entry hazard state and for the average hazard outside exclusion.

The effectiveness of exclusions is proportional to the degree that hazard varies over time. The potential benefit of using exclusions to manage seismic hazard relates to how seismicity is induced by the mining process. If seismicity is strongly related to blasting, the majority of seismic hazard can be mitigated using short exclusions after blasting. Conversely, if seismic hazard does not vary over time, there is no basis for a temporary exclusion since there is no difference between the average hazard with or without an exclusion.

When seismic hazard is assessed using the frequency and size distribution of recently recorded event data, there are inevitable differences between the true hazard state and the estimated hazard state. Figure 7 shows that estimating seismic hazard from recent data simplifies the true short-term fluctuations and there are biases when the hazard is changing. Seismic hazard is under-estimated when the true hazard is increasing and over-estimated when the true hazard is decreasing. This is important to recognise when considering evacuation protocols for large seismic events. The true seismic hazard can increase immediately after a large event, but there will be a time delay before the estimated seismic hazard recognises the change.

It is common to simplify the seismic process with a time-independent component that is superimposed with a time-dependent component. The time-independent component is often referred to as the background seismicity. These events are considered the result of a stationary Poisson process, meaning each event is 
independent of each other and there is a single long-term activity rate. The time-dependent component refers to triggered seismicity or short-term seismic responses which are often described temporally by the modified Omori law (MOL, Utsu 1961). Background seismicity is still variable in time and space, but over relatively longer time scales, related to general rates of extraction.

Defining re-entry time when seismic activity returns to the 'background level' after blasts is virtually ubiquitous in seismically active mines. This time is considered to represent when the effect of the blast on the seismic hazard has ceased. However, despite the seeming consensus, there is no widely accepted method for calculating the background activity rate, and there are some limitations with using this framework for exclusion and re-entry assessment. The background activity rate will also be heavily dependent on the sensitivity of the seismic network, which varies throughout a mine and over time.

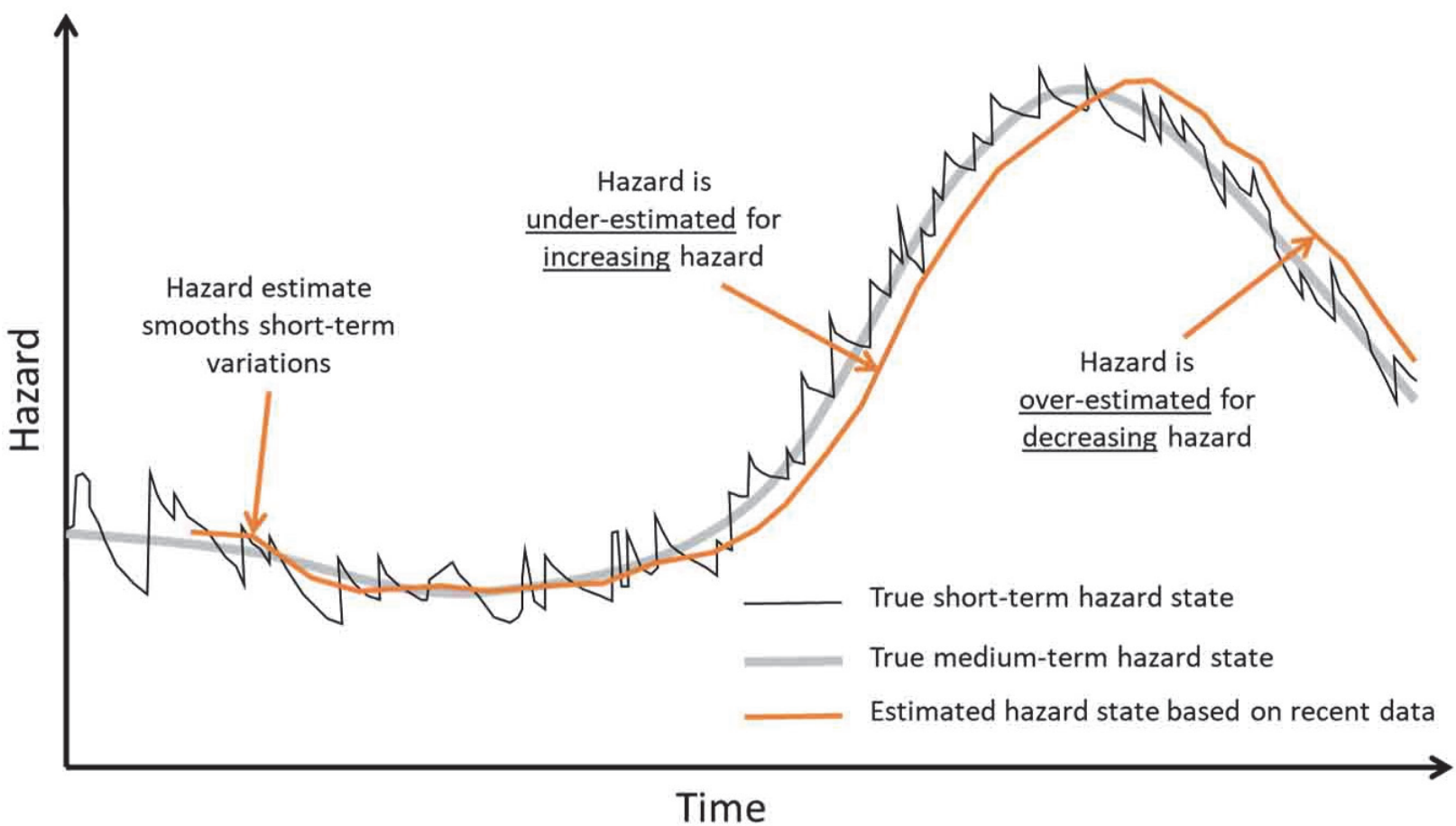

Figure 7 Illustration of the difference between true and estimated seismic hazard

If the background rate is the threshold for exclusion and re-entry, then by definition, a blast that triggers any response at all will have at least a short exclusion time. In practice, there will be cases where the background rate, even when superimposed with a minor response, represents a low hazard and there is no reason for an exclusion given the other seismic risk management controls in place.

The background activity rate does not necessarily correspond to the maximum tolerable seismic hazard for the relevant mining area. There are cases where there is more competent dynamic support installed where the background activity rate and seismic hazard is low.

If the threshold for re-entry is specified in terms of seismic hazard, the threshold can be adjusted depending on the other control measures in place and the excavation vulnerability in the given area. Background seismicity is a defensible starting point for the minimum requirements of risk management controls other than exclusions. The hazard posed by background seismicity cannot be controlled by short-term tactical exposure management and, therefore, must be managed by other controls, such as dynamic ground support systems.

The notion of a truly time-independent seismic rate is a simplification. If all short-term $\mathrm{MOL}$ seismic responses are removed from the dataset, there are still strong spatial and temporal variations in seismic activity that are not entirely random. Each event has the potential of triggering subsequent events. This potential increases with event magnitude. This is a key feature of the epidemic type aftershock sequence (ETAS, Ogata 1988) model, which is the current state-of-the-art in characterising earthquake occurrence. 
The current probabilistic seismic hazard assessment (PSHA) methodology (Kijko 2011) is generally only applied in the medium or long-term where there is sufficient data to define a reliable frequency-magnitude relationship (Gutenberg-Richter (GR) distribution). Effective re-entry assessment methods are good proxies for seismic hazard in the short-term using limited seismic data.

Short-term seismic hazard assessment became popular in South Africa in the early 1990s and typically involved interpreting trends in various seismic parameters over time. Perhaps the most widely known approach uses cumulative apparent volume (CAV) and energy index (EI) as an interpretive model of rock mass stability (van Aswegen \& Butler 1993). El is used as an approximation for the stress state of the rock mass, and CAV represents the amount of deformation. An elevated hazard would be indicated by an increase in stress state without deformation. Newcrest Mining Limited recently collaborated with the Australian Centre for Geomechanics to survey current seismic management practices around the world, and out of 30 seismically active mines surveyed, $56 \%$ use instability analysis at least on a monthly basis, while $20 \%$ said they use it daily (Potvin et al. 2018). Despite the wide use of instability analysis, there are few published examples where routine success has been demonstrated in estimating seismic hazard using this method.

Several other seismic source parameters have been used to assess seismic hazard for re-entry time scales (Alcott et al. 1998; Brown et al. 2015; Disley 2014; Poplawski 1997). It is rare for systematic back-analysis to be conducted for source parameter-based methods that includes an estimate of the effectiveness at estimating hazard and the number of false alarms. It is also important to be aware that seismic source parameter calculations often vary over time and between sites (Morkel 2017). The source calculations are heavily dependent on the limitations of the spatial coverage and recording capacities of the seismic network (Morkel et al. 2015). Many analysis techniques are suggested and documented, but it is important that there is independent verification of the effectiveness of the technique in different geological conditions and mining methods.

GR based seismic hazard calculations require the specification of an activity rate and relative size distribution (b-value). In most cases, the b-value is calculated based on a longer-term trend given data constraints. The MOL describes the activity rate of short-term seismic responses over time. Combined with a b-value characteristic of the time and location of the seismicity, Woodward (2015) provides an example of seismic hazard calculations for a short-term seismic response based on the MOL (Figure 8). The use of the MOL eliminates the previously mentioned time delay between true and estimated hazard since the activity rate is modelled rather than estimated with a moving time window.

A time-varying b-value is rarely used in seismic hazard calculations. However, in a study of the characteristics of mining-induced seismicity, Lasocki (2008) concluded that b-value was time-dependent. The temporal characteristics of $b$-value have been studied in other research fields. There are multiple cases where a drop in b-value has preceded rock mass failure in lab tests (Goebel et al. 2012; Main 1996; Meredith et al. 1990), numerical modelling (Kun et al. 2013), and earthquake case studies (Schorlemmer et al. 2005; Tormann et al. 2015). The probabilistic seismic hazard is highly sensitive to b-value. Gulia et al. (2016) compared three GR-based and two ETAS-based probabilistic seismic hazard calculations and found that using a time-varying b-value increased the estimated seismic hazard 50-fold, leading up to the L'Aquila earthquakes in Italy, 2009. In this case, using a time-varying b-value based on the most recent 100 events pushed the cost-benefit analysis in favour of evacuation. The validity and effectiveness of using a time-varying b-value in seismic hazard calculations has not yet been rigorously tested for mining seismicity. 


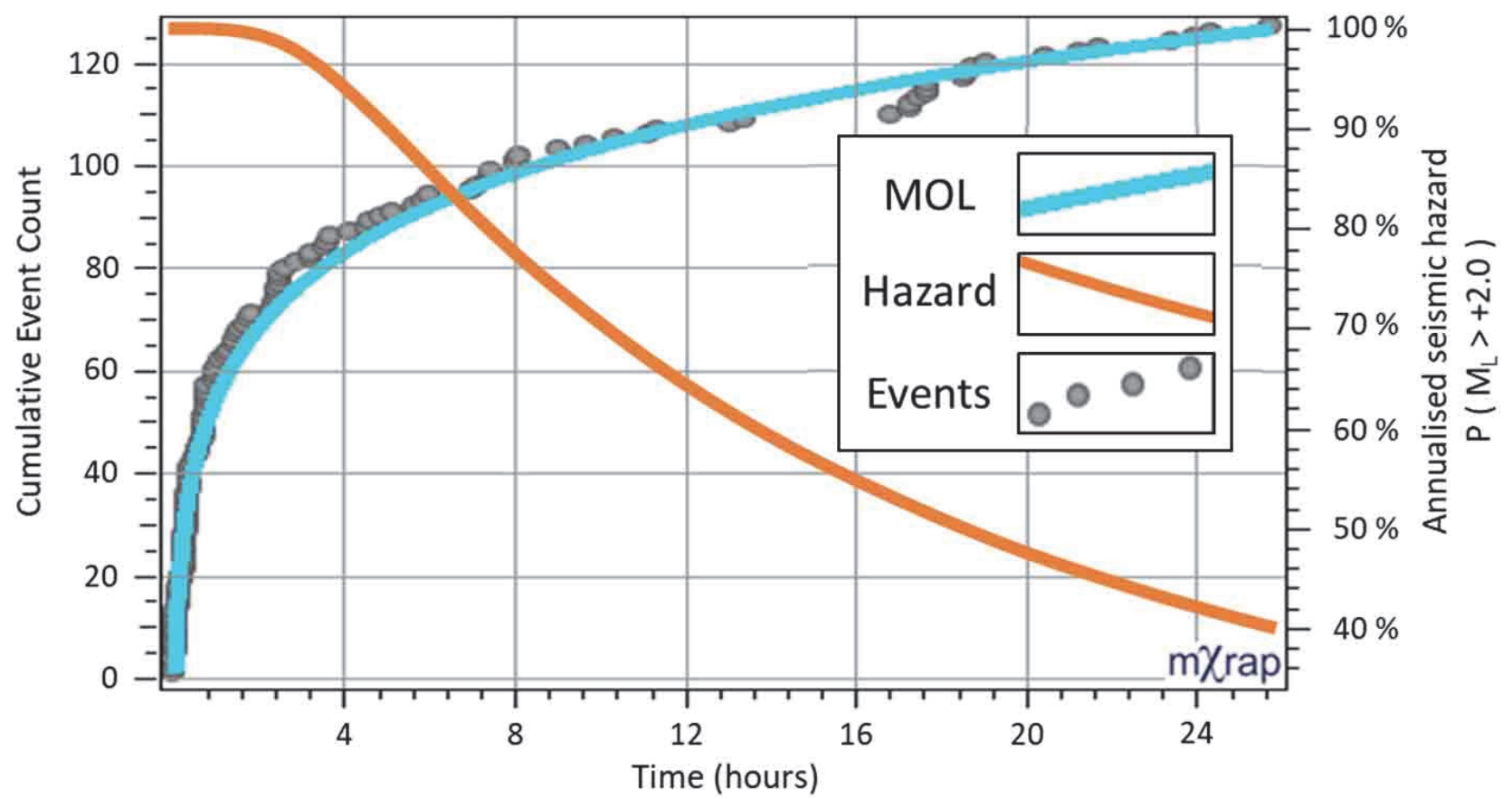

Figure 8 Probabilistic seismic hazard calculations for a response modelled with the MOL (Woodward 2015)

\section{$4 \quad$ Exposure management}

Exposure management generally consists of three separable types of protocols that dictate when to evacuate, where to exclude, and when to re-enter. The analysis techniques behind, and demonstrations of efficacy for, these protocols have been sparsely documented in the literature.

Evacuation typically occurs before blasting or after a large event. Mining operations may decide which blasts require an exclusion based on general principles such as depth, blast size, previous responses to firing and overall hazard. Occasionally there are specific protocols based on blast tonnage, where every firing over a certain size will require an exclusion, but it is rare for there to be specific assessment criteria behind these protocols. Evacuations following a large event are often defined for an arbitrarily chosen threshold event magnitude, usually based on previous experience and likelihood of aftershocks. Penney and Hills (2013) defined a magnitude threshold of $M_{\llcorner} 0.8$ for evacuation at the Tasmania mine based on the top $0.1 \%$ of events. Other seismic hazard indicators, such as elevated activity rate or EI/CAV trends, rarely trigger evacuations.

Exclusion areas are generally defined on a case-by-case basis, since they depend heavily on currently operating mining areas. It is sometimes easier to increase the exclusion area in places where there will be no operational effects. Some mines may define a spherical exclusion radius of 50-100 $\mathrm{m}$ from the blast location based on previous observations and spatial distribution of events.

Most re-entry decisions are based on the visual interpretation of seismicity, but there are some cases where specific rules are used to re-enter. Heal et al. (2005) defined re-entry when $90 \%$ of the cumulative energy had been released in the 24 hours following a blast. This was used to set exclusion times of blasts from retrospective analysis. Both the $90 \%$ and 24 -hour parameters are arbitrarily defined and can lead to very long re-entry times for weak responses to blasts.

Penney and Hills (2013) reviewed re-entry and exclusion practices at the Tasmania mine and established seismic parameter thresholds that must be met prior to re-entry. This approach reviewed the impact for production of implementing these rules from back-analysis, but did not consider if the rules resulted in improved risk management. The rules included a threshold to reset the exclusion clock if exceeded based on the background energy release and event rates. 
Vallejos and McKinnon (2009) developed a probabilistic framework for re-entry assessment that compares the current seismic response to previous responses. Morkel and Rossi-Rivera (2017) investigated the practical implementation of the Vallejos methodology and developed a stricter, quantitative definition of re-entry rules. This facilitated the systematic back-analysis of the Vallejos re-entry rules in terms of how successful it was at capturing large events within the exclusion time following blasts. The success was also quantified with respect to the average length of exclusion.

Figure 9 shows the results of a systematic back-analysis of three real-time re-entry assessment methods by Tierney and Morkel (2017). The Vallejos and McKinnon method was back-analysed using the approach described by Morkel and Rossi-Rivera (2017) and a database of short-term seismic responses modelled temporally with the MOL (Woodward \& Wesseloo 2015; Woodward et al. 2017, 2018). The two other real-time re-entry assessment methods used the event count and total energy within a moving time window.

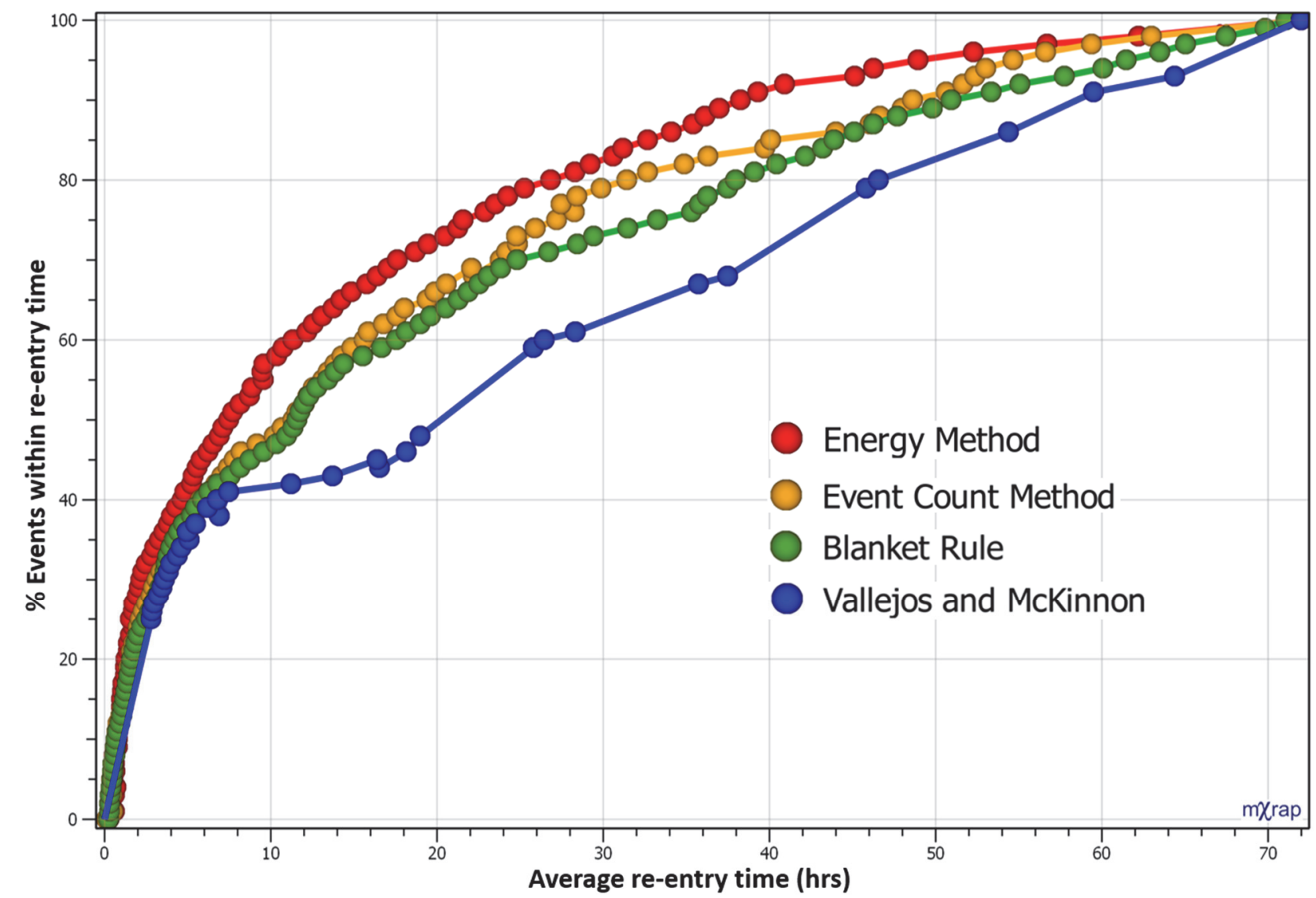

Figure 9 Comparison of optimised re-entry assessment methodologies for events greater than $M_{L}-0.5$ at a mine in Australia. Better re-entry methods will have a shorter exclusion time while capturing the same number of large events within the exclusion period (Tierney \& Morkel 2017).

Each re-entry method was optimised by maximising the number of significant events within the exclusion period and minimising the average period of exclusion. The optimised results for each real-time re-entry method were compared with a blanket exclusion period where the same re-entry time was used for each response. For the same number of significant events within exclusion, the energy method systematically resulted in shorter exclusion times and was, therefore, the most effective method investigated. A possible explanation for the success of the energy-based method (as a proxy for seismic hazard in the short-term) is that $b$-value is loosely captured by energy. As discussed already, seismic hazard is highly sensitive to b-value. 
The analysis by Tierney and Morkel (2017) is a good example of how the effect of specific exposure management procedures can be quantified. In this case, the analysis was limited to consider different forms of re-entry analysis. Risk-based design of exposure management protocols must consider the triggers for evacuation as well as the impact of different protocols for the area of exclusion.

\section{Conclusion}

Determining the appropriate evacuation triggers, the area of exclusion and the conditions for re-entry requires an iterative risk-based design methodology. An effective exposure management system would minimise the lost production time and minimise the seismic hazard during work periods. The design of exposure management protocols is only possible by correctly estimating the impact on safety risk and comparing with risk-based design acceptance criteria.

There is still a long way to go before the risk-based design framework for exposure management procedures is complete. This paper discussed several important areas for further research including:

- Incorporate the ALARP concept into the assessment of safety risk and the specification of risk-based design acceptance criteria.

- Consider methodologies for assessing the likelihood of adverse safety consequences from rockfalls.

- Investigate and compare the validity and effectiveness of different short-term seismic hazard assessment methods, including using a space- and time-varying b-value in hazard calculations.

- The estimation of the impact on safety risk of various exposure management procedures.

\section{Acknowledgement}

This paper is part of a research project on evidence-based design of exposure management protocols. Our thanks to IAMGOLD, Luossavaara-Kiirunavaara Aktiebolag (LKAB), and the Australian Centre for Geomechanics (ACG) for their support of this project. We also thank the members of the mXrap Consortium for their ongoing support.

\section{References}

Alcott, J, Kaiser, P \& Simser, B 1998, 'Use of microseismic source parameters for rockburst hazard assessment', Pure and Applied Geophysics, vol. 153, pp. 41-65.

Anderson, M \& Denkl, M 2010, 'The Heinrich accident triangle - too simplistic a model for HSE management in the 21st century?', Proceedings of the SPE International Conference on Health, Safety and Environment in Oil and Gas Exploration and Production, Society of Petroleum Engineers, Richardson, pp. 1062-1069.

Bohnenblust, H 1998, 'Risk-based decision making in the transportation sector', in RE Jorissen \& PJM Stallen (eds), Quantified Societal Risk and Policy Making, Springer, Basel.

Brown, L, Hudyma, M \& Turcotte, P 2015, 'Seismic hazard assessment using apparent stress ratio', in Y Potvin (ed.), Proceedings of the International Seminar on Design Methods in Underground Mining, Australian Centre for Geomechanics, Perth, pp. 123-134.

Cohen, J 2015, Managing Workplace Health and Safety, viewed 15 May 2015, http://sydney.edu.au/whs/activities/prioritise.shtml

Disley, N 2014, 'Seismic risk and hazard management at Kidd Mine', in M Hudyma \& Y Potvin (eds), Proceedings of the Seventh International Conference on Deep and High Stress Mining, Australian Centre for Geomechanics, Perth, pp. 107-121.

Finlay, P \& Fell, R 1997, 'Landslides: risk perception and acceptance', Canadian Geotechnical Journal, vol. 34, no. 2, pp. $169-188$.

Gallivan, S, Taxis, K, Franklin, B \& Barber, N 2008, 'Is the principle of a stable Heinrich ratio a myth?', Drug Safety, vol. 31, no. 8, pp. 1-6.

Goebel, T, Becker, T, Schorlemmer, D, Stanchits, S, Sammis, C, Rybacki, E \& Dresen, G 2012, 'Identifying fault heterogeneity through mapping spatial anomalies in acoustic emission statistics', Journal of Geophysical Research: Solid Earth, vol. 117, no. B3.

Gulia, L, Tormann, T, Wiemer, S, Herrmann, M \& Seif, S 2016, 'Short-term probabilistic earthquake risk assessment considering time-dependent b values', Geophysical Research Letters, vol. 43, no. 3, pp. 1100-1108.

Heal, D, Hudyma, M \& Vezina, F 2005, 'Seismic hazard at Agnico-Eagle's Laronde mine using MS-RAP', in JA Sag \& S Tessier (eds), Proceedings of the CIM Maintenance Engineering and Mine Operators Conference 2005, Canadian Institute of Mining, Sudbury.

Higson, D 1989, Risks to Individuals in NSW and in Australia as a Whole, Nuclear Safety Bureau, Yallambie.

Jonkman, S, van Gelder, P \& Vrijling, J 2003, 'An overview of quantitative risk measures for loss of life and economic damage', Journal of Hazardous Materials, vol. 99, no. 1, pp. 1-30. 
Joughin, N 2011, 'Engineering considerations in the tolerability of risk', The Journal of the Southern African Institute of Mining and Metallurgy, vol. 111, pp. 535-540.

Joughin, W, Jager, A, Nezomba, E \& Rwodzi, L 2012, 'A risk evaluation model for support design in Bushveld Complex underground mines: Part 1 - Description of the model', The Journal of the Southern African Institute of Mining and Metallurgy, vol. 112, pp. 83-94.

Joughin, W, Muaka, J, Mpunzi, P, Sewnun, D \& Wesseloo, J 2016, 'A risk-based approach to ground support design', in E Nordlund, T Jones \& A Eitzenberger (eds), Proceedings of the Eighth International Symposium on Ground Support in Mining and Underground Construction, Luleå University of Technology, Luleå.

Kijko, A 2011, 'Seismic hazard', in H Gupta (ed.), Encyclopedia of Solid Earth Geophysics, Springer, Basel.

Kirsch, P, Harris, J, Shi, M \& Cliff, D 2014, 'Reflections on mining and mortality', Australian Resources and Investment, vol. 8, no. 3, pp. 64-68.

Kun, F, Varga, I, Lennartz-Sassinek, S \& Main, I 2013, 'Approach to failure in porous granular materials under compression', Physical Review $E$, vol. 88, no. 6.

Lasocki, S 2008, 'Some unique statistical properties of the seismic process in mines', in Y Potvin, J Carter, A Dyskin \& R Jeffrey (eds), Proceedings of the First Southern Hemisphere International Rock Mechanics Symposium, Australian Centre for Geomechanics, Perth, pp. 667-678.

Main, I 1996, 'Statistical physics, seismogenesis, and seismic hazard', Reviews of Geophysics, vol. 34, no. 4, pp. $433-462$.

Manuele, F 2011, 'Reviewing Heinrich - dislodging two myths from the practice of safety', Professional Safety, October 2011, pp. 52-61.

Melchers, R 2001, 'On the ALARP approach to risk management', Reliability Engineering \& System Safety, vol. 71, no. 2, pp. $201-208$.

Meredith, P, Main, I \& Jones, C 1990, 'Temporal variations in seismicity during quasi-static and dynamic rock failure', Tectonophysics, vol. 175 , no. 1-3, pp. 249-268.

Morkel, IG \& Wessloo, J 2017, 'A technique to determine systematic shifts in microseismic databases', in J Wesseloo (ed.), Proceedings of the Eighth International Conference on Deep and High Stress Mining, Australian Centre for Geomechanics, Perth, pp. 105-116.

Morkel, IG \& Rossi-Rivera, P 2017, 'The implementation and quantification of the Vallejos and McKinnon re-entry methodology', in J Wesseloo (ed.), Proceedings of the Eighth International Conference on Deep and High Stress Mining, Australian Centre for Geomechanics, Perth, pp. 173-181.

Morkel, IG, Wesseloo, J \& Harris, P 2015, 'Highlighting and quantifying seismic data quality concerns', in P Dight (ed.), Proceedings of the Ninth International Symposium on Field Measurements in Geomechanics, Australian Centre for Geomechanics, Perth, pp. 539-549.

Ogata, Y 1988, 'Statistical models for earthquake occurrences and residual analysis for point processes', Journal of the American Statistical Association, vol. 83, no. 401, pp. 9-27.

Owen, M 2004, Exposure Model - Detailed Profiling and Quantification of the Exposure of Personnel to Geotechnical Hazards in Underground Mines, PhD thesis, The University of Western Australia, Perth.

Penney, A \& Hills, P 2013, 'Development of seismic heading re-entry and exclusion zones at the Tasmania mine', in A Malovichko \& D Malovichko (eds), Proceedings of the Eighth International Symposium on Rockbursts and Seismicity in Mines, Geophysical Survey of Russian Academy of Sciences, Obninsk, and Mining Institute of Ural Branch of Russian Academy of Sciences, Perm, pp. 447-459.

Poplawski, R 1997, 'Seismic source parameters and rockburst hazard at Mt. Charlotte Mine', International Journal of Rock Mechanics and Mining Sciences, vol. 34, pp. 1213-1228.

Potvin, Y, Wesseloo, J, Woodward, K, Morkel, I \& Tierney, S 2018, Seismic Risk Management Practices in Underground Mines, viewed 3 December 2018, https://www.acg.uwa.edu.au/srmp

Safe Work Australia 2016, 'Work-related traumatic injury fatalities', Comparative Performance Monitoring Report, 18th edn, Australia, Safe Work Australia, Canberra.

Schorlemmer, D, Wiemer, S \& Wyss, M 2005, 'Variations in earthquake-size distribution across different stress regimes', Nature, vol. 437, no. 7058 , pp. 539.

Sherratt, F 2014, 'Exploring 'zero target' safety programmes in the UK construction industry', Construction Management and Economics, vol. 32, no. 7-8, pp. 737-748.

Stacey, T, Terbrugge, P \& Wesseloo, J 2007, 'Risk as a rock engineering design criterion', in Y Potvin, T Stacey \& J Hadjigeorgiou (eds), Challenges in Deep and High Stress Mining, Australian Centre for Geomechanics, Perth, pp. 19-26.

Talbot, J 2014, ALARP (As Low As Reasonably Practicable), Jakeman Business Solutions, Symonston.

Terbrugge, P, Wesseloo, J, Venter, J \& Steffen, O 2006, 'A risk consequence approach to open pit slope design', The Journal of the Southern African Institute of Mining and Metallurgy, vol. 106, pp. 503-514.

Tierney, S \& Morkel, IG 2017, 'The optimisation and comparison of re-entry assessment methodologies for use in seismically active mines', in J Wesseloo (ed.), Proceedings of the Eighth International Conference on Deep and High Stress Mining, Australian Centre for Geomechanics, Perth, pp. 183-196.

Tormann, T, Enescu, B, Woessner, J \& Wiemer, S 2015, 'Randomness of megathrust earthquakes implied by rapid stress recovery after the Japan earthquake', Nature Geoscience, vol. 8, no. 2, pp. 152.

Utsu, T 1961, 'A statistical study of the occurrence of aftershocks', Geophysical Magazine, vol. 30, no. 4.

Vallejos, J \& McKinnon, S 2009, 'Re-entry protocols for seismically active mines using statistical analysis of aftershock sequences', in M Diederichs \& G Grasselli (eds), Proceedings of the Third CANUS Rock Mechanics Symposium, Canadian Rock Mechanics Association, p. 143. 
van Aswegen, G \& Butler, A 1993, 'Application of quantitative seismology in SA gold mines', in R Young (ed.), Proceedings of the Third International Symposium on Rockbursts and Seismicity in Mines, A.A. Balkema, Rotterdam, pp. 261-266.

Vrijling, J, van Hengel, W \& Houben, R 1995, 'A framework for risk evaluation', Journal of Hazardous Materials, vol. 43, pp. $245-261$.

Vrijling, J, van Hengel, W \& Houben, R 1998, 'Acceptable risk as a basis for design', Reliability Engineering and System Safety, vol. 59, pp. 141-150.

Wentzel, N, Pooley, T \& Sheriff, B 2012, 'Is there harm in zero?', Proceedings of the SPE Asia Pacific Oil \& Gas Conference and Exhibition 2012, vol. 1, Society of Petroleum Engineers, Richardson.

Wesseloo, J \& Joughin, W 2019, internal document, Australian Centre for Geomechanics, Perth.

Woodward, KR 2015, Identification and Delineation of Mining Induced Seismic Responses, PhD thesis, The University of Western Australia, Perth.

Woodward, KR \& Wesseloo, J 2015, 'Observed spatial and temporal behaviour of seismic rock mass response to blasting', Journal of the Southern African Institute of Mining and Metallurgy, vol. 115, no. 11, pp. 1044-1056.

Woodward, KR, Wesseloo, J \& Potvin, Y 2017, 'Temporal delineation and quantification of short term clustered mining seismicity', Pure and Applied Geophysics, vol. 174, no. 7, pp. 2581-2599.

Woodward, KR, Wesseloo, J \& Potvin, Y 2018, 'A spatially focused clustering methodology for mining seismicity', Engineering Geology, vol. 232, pp. 104-113.

Yudkowsky, E 2008, 'Cognitive biases potentially affecting judgment of global risks', Global Catastrophic Risks, vol. 1, no. 86. 